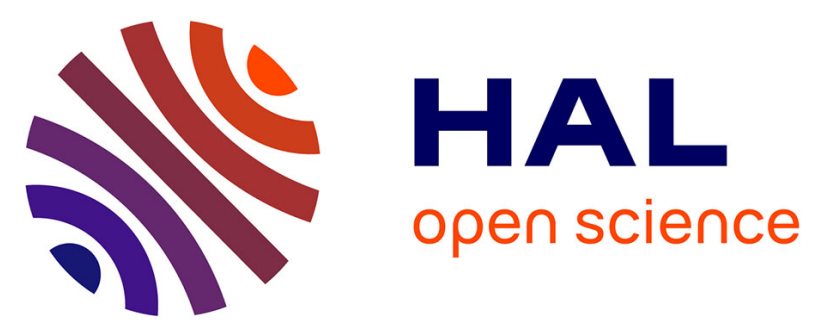

\title{
Energy-dispersive X-ray absorption spectroscopy at LNLS: investigation on strongly correlated metal oxides
} Julio C. Cezar, Narcizo M. Souza-Neto, Cinthia Piamonteze, Edilson Tamura, Flavio Garcia, Edson J. Carvalho, Régis T. Neueschwander, Aline Y. Ramos, Hélio C. N. Tolentino, Alberto Caneiro, et al.

\section{To cite this version:}

Julio C. Cezar, Narcizo M. Souza-Neto, Cinthia Piamonteze, Edilson Tamura, Flavio Garcia, et al.. Energy-dispersive X-ray absorption spectroscopy at LNLS: investigation on strongly correlated metal oxides. Journal of Synchrotron Radiation, 2010, 17, pp.93-102. 10.1107/S0909049509041119 . hal00443730

\section{HAL Id: hal-00443730 \\ https://hal.science/hal-00443730}

Submitted on 4 Jan 2010

HAL is a multi-disciplinary open access archive for the deposit and dissemination of scientific research documents, whether they are published or not. The documents may come from teaching and research institutions in France or abroad, or from public or private research centers.
L'archive ouverte pluridisciplinaire HAL, est destinée au dépôt et à la diffusion de documents scientifiques de niveau recherche, publiés ou non, émanant des établissements d'enseignement et de recherche français ou étrangers, des laboratoires publics ou privés. 
Journal of

Synchrotron

Radiation

ISSN 0909-0495

Received 16 May 2009

Accepted 8 October 2009

C 2010 International Union of Crystallography

Printed in Singapore - all rights reserved

\section{Energy-dispersive X-ray absorption spectroscopy at LNLS: investigation on strongly correlated metal oxides}

\author{
Júlio C. Cezar, ${ }^{\mathrm{a}} \neq$ Narcizo M. Souza-Neto, ${ }^{\mathrm{a}} \oplus$ Cínthia Piamonteze, ${ }^{\mathrm{a}} \S$ \\ Edilson Tamura, ${ }^{a} \neq \ddagger$ Flávio Garcia, ${ }^{a}$ Edson J. Carvalho, ${ }^{a}$ Régis T. Neueschwander, ${ }^{a}$ \\ Aline Y. Ramos, ${ }^{\text {b }}$ Hélio C. N. Tolentino, ${ }^{\text {b* }}$ Alberto Caneiro, ${ }^{\mathrm{c}}$ Nestor E. Massa, ${ }^{\mathrm{d}}$ \\ Maria Jesus Martinez-Lope, ${ }^{e}$ Jose Antonio Alonso ${ }^{e}$ and Jean-Paul Itié ${ }^{f}$ \\ aLaboratório Nacional de Luz Síncrotron - LNLS, CP6192, 13084-971 Campinas, SP, Brazil, \\ ${ }^{\mathbf{b}}$ Institut Néel, CNRS and UJF, BP 166, 38042 Grenoble, France, ${ }^{\mathbf{c} C o m i s i o n ~ N a c i o n a l ~ d e ~ E n e r g i a ~}$ \\ Atómica, Centro Atomico Bariloche and Instituto Balseiro, Bariloche, Argentina, dLaboratorio \\ Nacional de Investigacion y Servicios en Espectroscopia Optica - CEQUINOR, Universidad \\ Nacional de La Plata, La Plata, Argentina, e Instituto de Ciencia de Materiales de Madrid, CSIC, \\ Cantoblanco, Madrid, Spain, and 'Synchrotron SOLEIL, L'Orme des Merisiers, Saint-Aubin, France. \\ E-mail: helio.tolentino@grenoble.cnrs.fr
}

An energy-dispersive X-ray absorption spectroscopy beamline mainly dedicated to X-ray magnetic circular dichroism (XMCD) and material science under extreme conditions has been implemented in a bending-magnet port at the Brazilian Synchrotron Light Laboratory. Here the beamline technical characteristics are described, including the most important aspects of the mechanics, optical elements and detection set-up. The beamline performance is then illustrated through two case studies on strongly correlated transition metal oxides: an XMCD insight into the modifications of the magnetic properties of Cr-doped manganites and the structural deformation in nickel perovskites under high applied pressure.

Keywords: XAS; XMCD; high pressure.

\section{Introduction}

The Brazilian Synchrotron Light Laboratory (LNLS, Campinas, Brazil) runs a $1.37 \mathrm{GeV}$ electron storage ring (Rodrigues et al., 1998) endowed with a $120 \mathrm{MeV}$ LINAC and $500 \mathrm{MeV}$ booster injector system (Lin et al., 2001) operating at a maximum current of $300 \mathrm{~mA}$. The energy-dispersive X-ray absorption spectroscopy (EDXAS) beamline described here is installed in a bending-magnet port and was delivered to the users in 2005. The peculiar capability of an EDXAS spectrometer is to collect full absorption spectra through a parallel detection scheme over an extended range of photon energies without any mechanical movement (Phizackerley et al., 1983; Dartyge et al., 1986). The parallel detection, combining good spatial resolution, ability to handle the high flux of photons (of the order $10^{11}$ photons $\mathrm{s}^{-1}$ ) and fast readout times makes EDXAS specially suited to detecting very weak signals in

¥ Present address: European Synchrotron Radiation Facility, Grenoble, France.

T Present address: Advanced Photon Source, Argonne National Laboratory, Argonne, USA.

$\S$ Present address: Swiss Light Source, Paul Scherrer Institut, Villegen PSI, Switzerland.

$\$$ Present address: Condutiva Tecnologia, Campinas, SP, Brazil.
XANES (X-ray absorption near-edge spectroscopy) and XMCD (X-ray magnetic circular dichroism) experiments (Baudelet et al., 1991; Pizzini et al., 1995), for tracking timedependent evolution of chemical reactions (Tourillon et al., 1986) and attaining nanosecond resolution (Bonfim et al., 1998). Owing to the absence of moving parts during the acquisition, the beam in an EDXAS set-up is inherently highly stable in both energy scale and spatial position. These features, along with the intrinsic focused beam and the parallel detection, enable high-pressure studies using diamond anvil cells (Itié et al., 1989; Pascarelli et al., 2004), even when associated with XMCD experiments (Mathon et al., 2004), and the possibility of probing femtometer-scale atomic displacements (Pettifer et al., 2005).

From 2005 onwards, many experimental facilities have been developed at the LNLS EDXAS beamline; for example, a halogen-lamp-based furnace for controlled thermal treatments and study of dynamical processes (Meneses et al., 2006). This furnace made possible the observation of $\mathrm{NiO}$ nanoparticles undergoing a phase transformation (amorphous to nanocrystal) during annealing (Meneses et al., 2007). A reactor chamber (Bernardi et al., 2007) was used to monitor the chemical environment of $\mathrm{Pt}$ atoms in a catalytic reaction of 
$\mathrm{Pt}_{x} \mathrm{Pd}_{1-x}$ nanoparticles (Bernardi et al., 2009). Both chambers are usually coupled to a mass spectrometer for residual gas analysis. An electrochemical cell for in situ voltammetry analysis was employed to study the oxy-redoxy reaction of methanol on carbon-supported Pt-Ru nanocatalysts (Godoi et al., 2009). High-pressures studies have been reported in two different pressure domains, one up to $1.2 \mathrm{GPa}$ using a $\mathrm{B} 4 \mathrm{C}$ anvil cell (Ferreira et al., 2009) and another up to $15 \mathrm{GPa}$ using a perforated diamond cell (Ramos et al., 2007). A set-up for grazing-incidence $\mathrm{X}$-ray resonant magnetic scattering (SouzaNeto et al., 2006) and the use of a closed-cycle He cryostat associated with a $0.9 \mathrm{~T}$ permanent magnet for XMCD experiments (Azimonte et al., 2007; Stewart et al., 2007) have also been reported. Many other experiments on magnetic materials will soon become feasible with the forthcoming installation of a $6.5 \mathrm{~T}$ superconducting magnet. As may be noted, the LNLS EDXAS beamline covers the traditional fields of similar existing facilities around the world. Some strengths and limitations when compared with these facilities will be discussed throughout the paper.

We will give here an extensive description of the EDXAS beamline that includes the optical elements, some important aspects of the mechanics, and the detector system. Then, the beamline performance is illustrated by two studies related to main instrumental developments of the beamline: an XMCD set-up is used to study the magnetic properties of Cr-doped manganites and a diamond anvil cell is used to study the structural changes of nickel perovskites under high pressure.

\section{The energy-dispersive X-ray spectrometer}

The energy-dispersive X-ray spectrometer uses a bent $\mathrm{Si}$ crystal monochromator to provide a continuous energy variation of the Bragg-reflected photons (Fig. 1). The monochromator is bent in such a way that the incidence angle of the white synchrotron light beam changes continuously along the crystal, thus selecting a bandwidth of hundreds of $\mathrm{eV}$ and focusing it horizontally at the sample position. There is a correlation between the photon energy and the horizontal position at the detector. A complete X-ray absorption spectrum is collected in a single detector's readout. Vertical focusing as well as harmonic rejection are accomplished by a bendable mirror before the Si crystal.

\subsection{The energy-dispersive optics}

The source-to-crystal and crystal-to-focus distances in Fig. 1 are represented by the variables $p$ and $q$, respectively. The average curvature radius $R$ is related to $p$ and $q$ by $1 / p+1 / q=$ $2 /\left(R \sin \theta_{\mathrm{B}}\right)$, where $\theta_{\mathrm{B}}$ is the average Bragg angle of reflection. The photon energy $E$ depends on the Bragg angle $\theta_{\mathrm{B}}$ according to $E[\mathrm{eV}]=12398 /\left(2 d[\AA] \sin \theta_{\mathrm{B}}\right)$, where $d$ is the $\mathrm{Si}$ crystal lattice spacing. The angle changes along the illuminated crystal length $L$, yielding a relative energy bandwidth $\Delta E / E=\Delta \theta \cot \theta_{\mathrm{B}}$, with $\Delta \theta=L / R\left(1-R \sin \theta_{\mathrm{B}} / p\right)$. The full energy bandwidth is focused at the sample. The energy resolution depends essentially on the intrinsic crystal band-pass,

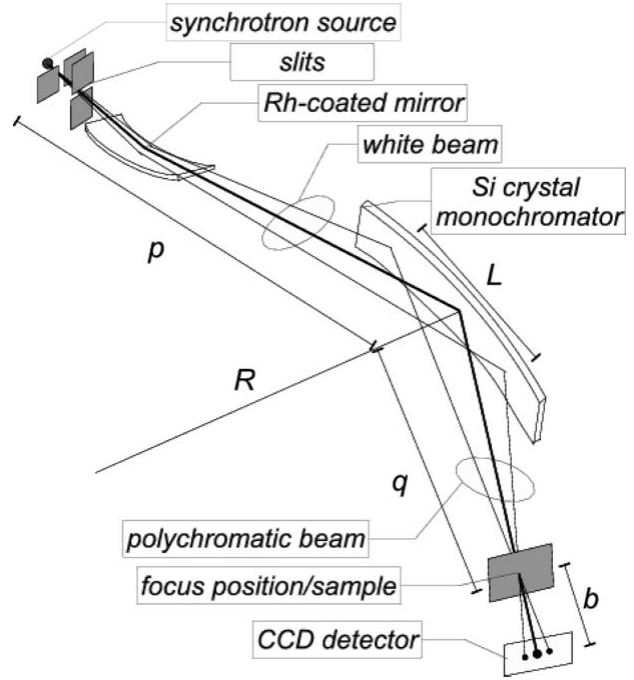

Figure 1

Schematic view of the EDXAS set-up showing the synchrotron source, the vertically focusing mirror, the bent crystal monochromator and the detector. The sample is positioned at the focus of the monochromator. The photon energy-direction correlation is transformed into an energyposition correlation along the horizontal axis of the detector.

spatial resolution of the detector and horizontal source dimension. In order to optimize energy resolution the detector is normally placed at a distance $b$ from the sample such that $b \simeq q$ (Tolentino et al., 1988). As can be noted, energy bandpass and resolution depend on intrinsic as well as on extrinsic parameters, like $L, R$ and $p$.

The EDXAS beamline at LNLS is installed on a bendingmagnetic port (Fig. 2). The horizontal and vertical dimensions (FWHM) of the source are $s_{x}=750 \mu \mathrm{m}$ and $s_{y}=168 \mu \mathrm{m}$, respectively. The source-to-crystal distance was chosen as short as possible $(p=9.75 \mathrm{~m})$ to provide a large beam horizontal acceptance by the bent crystal. This acceptance is limited to a maximum of $4.8 \mathrm{mrad}$ by the detector horizontal acceptance. The crystal-to-detector distance is adjustable between 1 and $3.5 \mathrm{~m}$. The $2 \theta$ arm can be adjusted to angles between 56 and $16^{\circ}$, providing, in principle, an energy range from 4 to $14 \mathrm{keV}$ with the $\mathrm{Si}(111)$ crystal. A compromise between energy band-pass, energy resolution and sample position has to be found for each experimental situation. The energy band-pass and energy resolution for typical $q$ values and at $b \simeq q$ are presented in Figs. 3(a) and 3(b), respectively. The dotted lines in Fig. 3(a) represent the band-pass limited by the crystal size while the continuous ones give the band-pass limited by the detector window when $b \simeq q$. One may set the distance $b$ smaller than $q$ to gain band-pass with the drawback of losing energy resolution.

The lowest attainable energy depends on the sample and on its environment, especially when extreme conditions are required. Up to now, the lowest energy achieved in a real experiment is the one reported here for Cr-doped $\mathrm{LaMnO}_{3}$. The $\mathrm{La} L_{2}$ edge $(5891 \mathrm{eV})$ is easily accessible owing to its high absorption cross section. However, the strong attenuation imposed by La atoms, whose $L_{3}$ and $L_{2}$ edges are at $5483 \mathrm{eV}$ and $5891 \mathrm{eV}$, respectively, makes experiments at the $\mathrm{Cr} K$ edge 


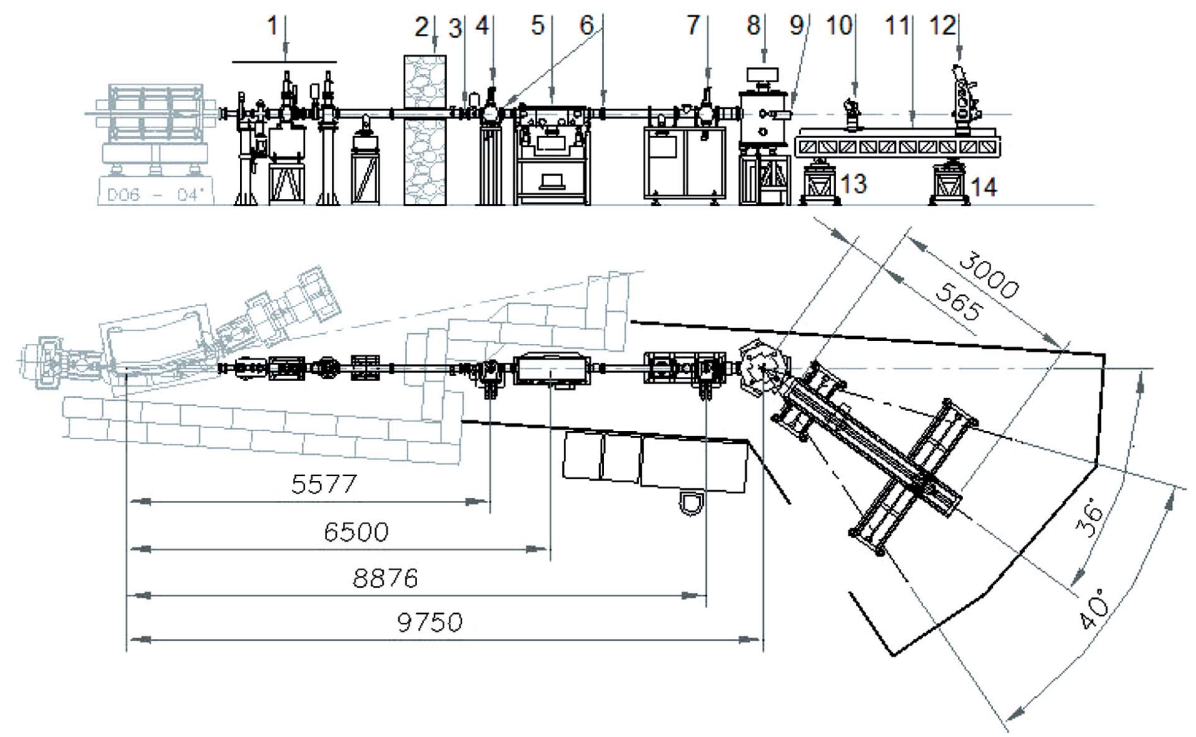

Figure 2

Layout of the EDXAS beamline installed in a bending-magnet port at LNLS. The sequence indicates: (1) front-end, (2) wall shield, (3) cooled Be window, (4) cooled slits, (5) Rh-coated mirror, (6) two Be windows, (7) cooled slits, (8) monochromator, (9) beam shutter and large Be window, (10) sample holder, (11) optical bench, (12) modified CCD detector, (13) short linear stage and (14) long linear stage. Distances are in millimeters.

very challenging, especially when only $5 \%$ of $\mathrm{Cr}$ atoms substitute for $\mathrm{Mn}$ atoms in the perovskite structure.

The monochromator bender mechanism developed at LNLS is detailed elsewhere (Neuenschwander \& Tolentino, 2004). Such a bender is supported by four columns and assembled on top of a three-circle goniometer with the center of the crystal's front face coincident with the three-circle confusion sphere (Fig. 4). The main circle is used to set the energy, and the others are used for the preliminary alignment procedure. The vacuum vessel allows future installation of a second bender, with a different crystal reflection plane, to extend the covered energy range. All elements inside the vessel are UHVcompatible and isolated from the beamline by a $0.125 \mathrm{~mm}$ Be entrance window. The beam-output window is a flat $385 \mathrm{~mm}$-long $10 \mathrm{~mm}$-wide $0.125 \mathrm{~mm}$-thick $\mathrm{Be}$ foil brazed on a

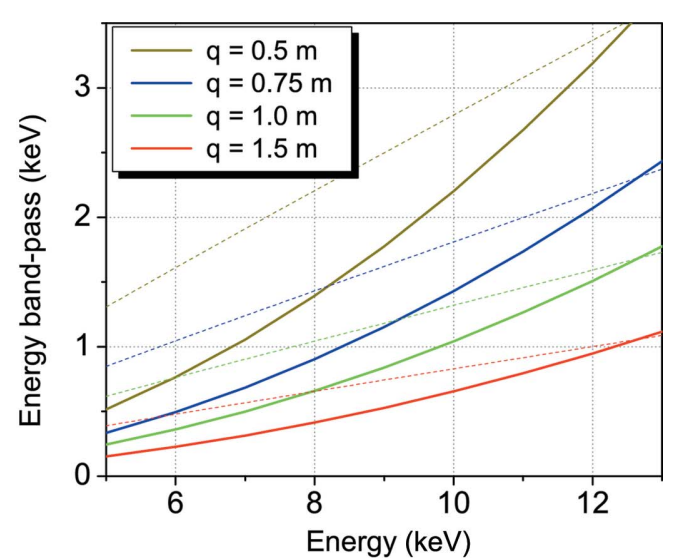

(a)

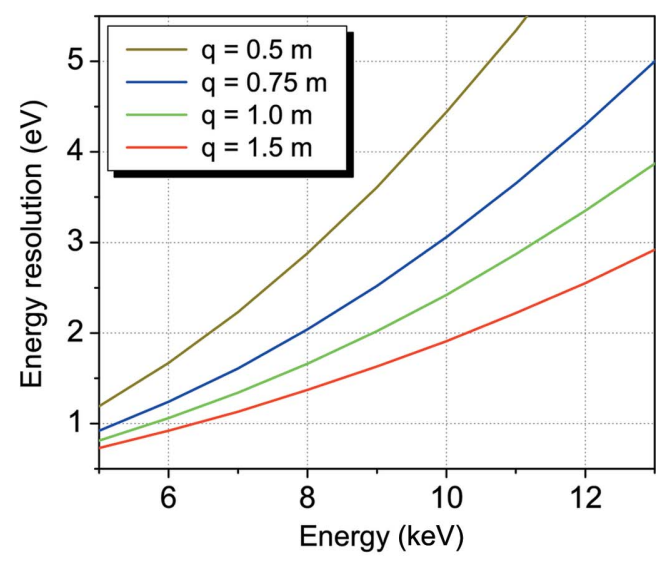

(b)

Figure 3

Energy band-pass ( $a$ ) and energy resolution $(b)$ for typical $q$ values and at $b \simeq q$ in the case of a $\operatorname{Si}(111)$ bent crystal. The dotted lines in $(a)$ represent the band-pass limited by the crystal size while the continuous ones give the band-pass limited by the detector window when $b \simeq q$. stainless steel flange, equipped with a pneumatic shutter. A $3401 \mathrm{~s}^{-1}$ ion pump, assembled on the chamber, provides vacuum conditions without any vibration. The monochromator operates with a base pressure of $5.0 \times 10^{-8} \mathrm{mbar}$ that prevents crystal surface degradation.

The curvature radius $R$ of the $\mathrm{Si}$ crystal is given by two independent torques imposed by linear actuators at each extremity. The mechanism is designed to bend a $300 \mathrm{~mm} \times$ $20 \mathrm{~mm}$ rectangular crystal from $8 \mathrm{~m}$ to $3 \mathrm{~m}$, given by a displacement of $2.3 \mathrm{~mm}$ of the actuator, without displacement of the central part (Neuenschwander \& Tolentino, 2004). The combination of the two independent torques at each crystal extremity provides a way to significantly reduce spherical

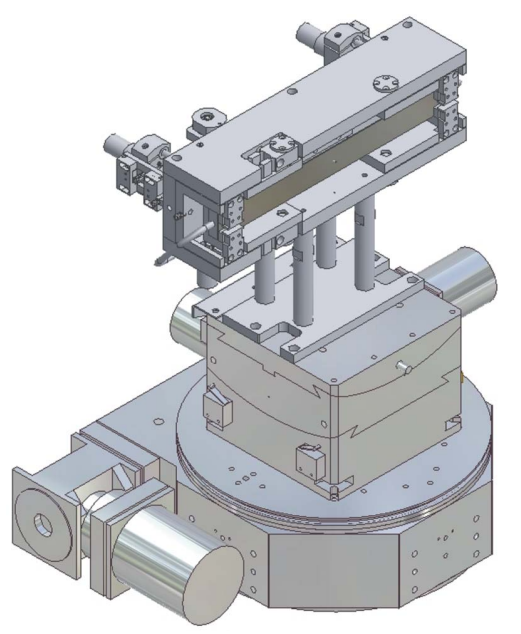

Figure 4

Bender mechanism for the crystal monochromator on top of the threecircle goniometer. The main circle is used to set the energy, and the others are used during the preliminary alignment procedure. 
aberrations. One of the frames that transmit torque to the crystal is assembled on an elastic link enabling twist correction. A $0.25 \mathrm{~mm}$ eccentric twist stroke is essential for further focus optimization.

Fig. 5 presents a comparison in a situation where the aberrations are minimized with equal applied torques on both extremities (Fig. 5a) and the same situation where the two torques are optimized independently (Fig. $5 b$ ). The measurements were accomplished around the Co $K$ edge $(7709 \mathrm{eV})$ by scanning a $20 \mu \mathrm{m}$ slit laterally through the focus position, while collecting images in the CCD-based detector. The vertical axis corresponds to the slit relative translation and the horizontal axis to the photon energy. The beam size (FWHM) at each energy is $125 \mu \mathrm{m}$ for both situations, given by source size $(750 \mu \mathrm{m})$ and a demagnification $(q / p)$ of $1 / 6$. However, one can observe that the full band-pass is spread over $350 \mu \mathrm{m}$ when symmetric torques are applied and the focus spot is

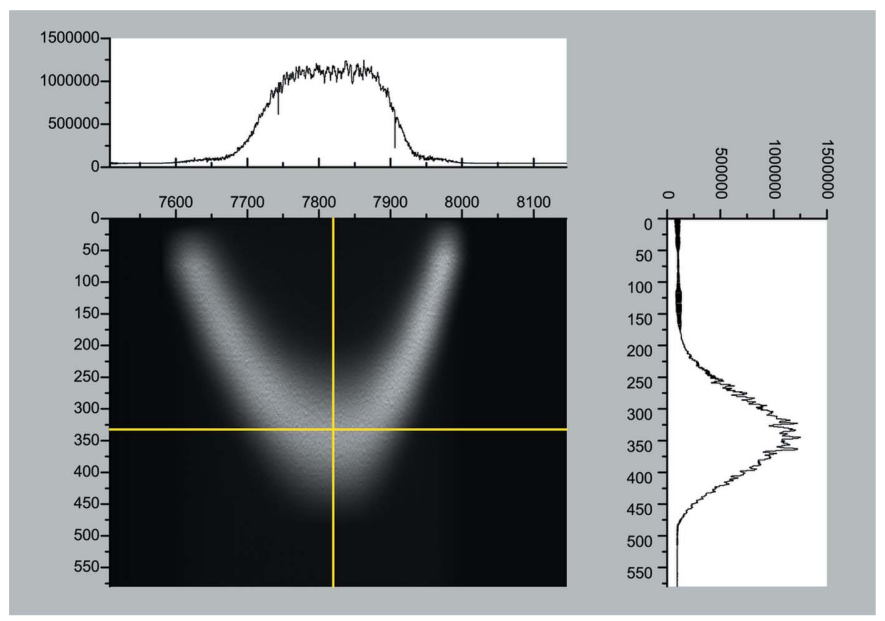

(a)

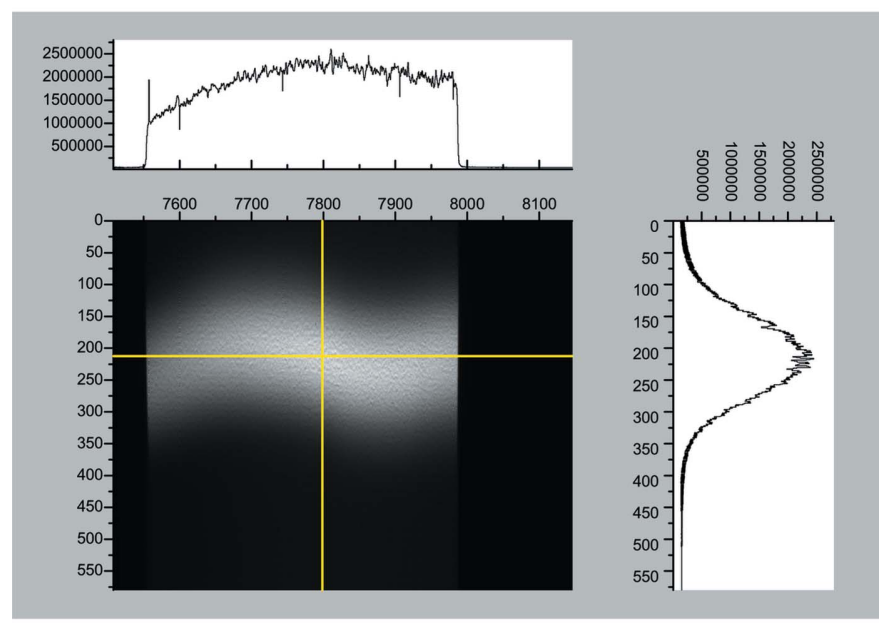

(b)

Figure 5

Focus point: comparison of a situation where the spherical optical aberration is minimized with the same applied torque on both extremities (a) and the same situation where the two torques are optimized separately $(b)$. The vertical axis corresponds to the slit translation and the horizontal axis to the photon energy. The top and side curves are image cross sections. dominated by the spherical aberrations. On the other hand, when the focus is optimized, by applying asymmetric torques the aberration is reduced to less than $30 \mu \mathrm{m}$ and the full bandpass is focused within $150 \mu \mathrm{m}$. The horizontal beam size that should be considered is in fact about $250 \mu \mathrm{m}$ (full beam) when the tails are included to have more than $95 \%$ of the photons. The remaining S-shape aberration could be further reduced by a small correction in the rectangular crystal shape. This correction has not been implemented at the EDXAS beamline because the focused beam is largely limited by the bendingmagnet source size, and demagnification and aberrations contribute less than $10 \%$ to the beam size. For a horizontal beam slit down to the full beam, the tails of the Gaussianshaped beam have negligible contribution to the intensity.

Vertical focusing and harmonic rejection are provided by an $800 \mathrm{~mm}$-long Rh-coated glass mirror at $6.5 \mathrm{~m}$ from the source (Neuenschwander \& Tavares, 2001). The mirror is separated from the front-end and the monochromator by two $125 \mu \mathrm{m}$ thick Be windows and operates with a base pressure of $1.0 \times$ $10^{-8}$ mbar. The bending mechanism yields at the sample a vertically focused beam (FWHM) of about $200 \mu \mathrm{m}$, limited by the storage ring vertical emittance and a demagnification close to $1: 1$. The mirror provides a cut-off energy of about $15 \mathrm{keV}$ at a grazing angle of $4 \mathrm{mrad}$, the typical working angle for most experiments. At photon energies lower than $7 \mathrm{keV}$ and strongly absorbing samples, the grazing angle has to be increased up to $4.5 \mathrm{mrad}$ to reduce the cut-off energy to about $12 \mathrm{keV}$. A second small mirror is normally installed, between the sample and the detector, for further reducing harmonic contamination.

The bending-magnet source, front-end and photon flux characteristics have already been discussed elsewhere (Tolentino et al., 2005b). In normal operation conditions, the photon flux in a spot of $250 \mu \mathrm{m} \times 200 \mu \mathrm{m}$ at the sample is of the order of $1.0 \times 10^{11}$ photons s ${ }^{-1}$ at $8 \mathrm{keV}$. This corresponds to the $\mathrm{Si}(111)$ crystal monochromator (bandwidth of $0.013 \%$ ) accepting a horizontal divergence of $4 \mathrm{mrad}$ and the storage ring working with $200 \mathrm{~mA}$. The photon flux can be improved by a factor of two at $8 \mathrm{keV}$ by pumping along the beam path. Pumping the beam path is compulsory when working at energies lower than $7 \mathrm{keV}$. For instance, at the $\mathrm{Cr} K$ edge $(5898 \mathrm{eV})$ the photon flux is improved by one order of magnitude, from $6.0 \times 10^{10}$ photons $\mathrm{s}^{-1}$ up to $4.0 \times$ $10^{11}$ photons s $^{-1}$. It must be noted that the beam path in the air is the most severe factor of attenuation and not the four $125 \mu \mathrm{m}$-thick $\mathrm{Be}$ windows, whose contribution to the attenuation is only $20 \%$ (Tolentino et al., 2005b). Such flux is smaller by a factor of ten when compared with a similar facility, the ODE beamline at Soleil, and by four to five orders of magnitude when compared with the undulator beamline ID24 at ESRF.

\subsection{The CCD-based detection scheme}

The high photon flux is handled by a modified CCD camera that uses a phosphor screen to convert X-rays into visible light (Fig. 6). The cryogenically cooled CCD camera system has a 


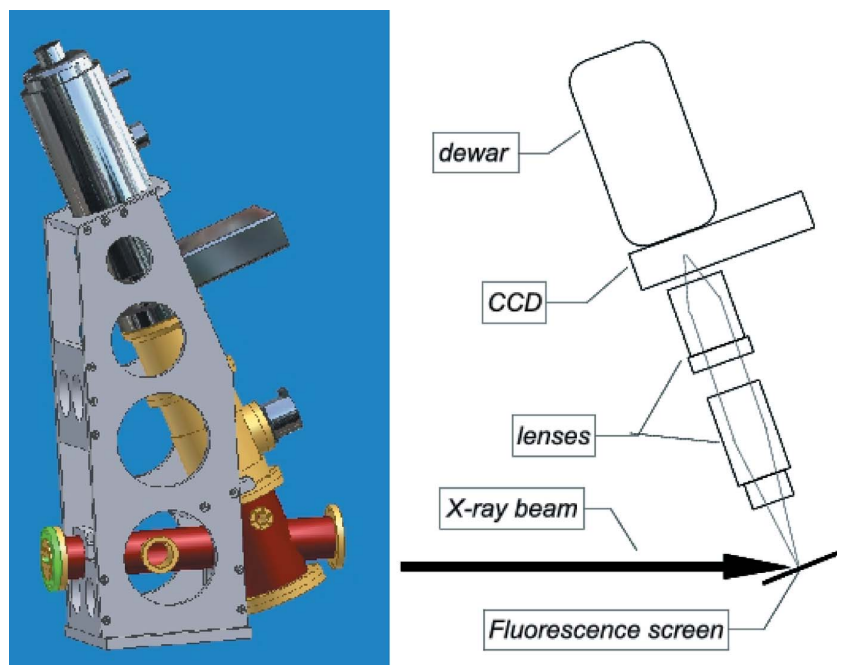

Figure 6

Schematic view of the modified CCD detector. X-rays are converted into fluorescence photons on a screen inclined at a grazing angle of $20^{\circ}$ relative to the beam. In such a case, the beam is spread over a large area along the beam direction on the screen and saturation of the CCD detector is avoided. The perpendicular direction is used for the energyposition correlation. Lenses are used to collect as many fluorescence photons as possible and to focus them into the CCD. A demagnification factor of $1: 1.75$ is used.

front-illuminated scientific grade $1 \mathrm{CCD}$ of $1340 \times 1300$ pixels. Each pixel is $20 \mu \mathrm{m} \times 20 \mu \mathrm{m}$ providing an image area of $26.8 \mathrm{~mm} \times 26.0 \mathrm{~mm}$. A $20 \mu \mathrm{m}$-thick $\mathrm{P} 43\left(\mathrm{Gd}_{2} \mathrm{O}_{3} \mathrm{~S}: \mathrm{Tb}\right)$ fluorescence screen, optimized for $8 \mathrm{keV} \mathrm{X-rays} \mathrm{(Koch,} \mathrm{1994),}$ receives the incoming beam at an angle of $20^{\circ}$. A set of lenses guides the light from the screen to the CCD detector with a demagnification factor of $1: 1.75$, so that the projected pixel size and useful area at the fluorescence screen are $35 \mu \mathrm{m} \times$ $35 \mu \mathrm{m}$ and $46.9 \mathrm{~mm} \times 46.5 \mathrm{~mm}$, respectively. Actually, the spatial resolution measured in the energy dispersion direction is about 2.5 to 3 times larger than the projected pixel, i.e. $100 \mu \mathrm{m}$, owing to the penetration and blurring into the fluorescence screen. To improve spatial resolution, which turns out to be the limiting factor in the overall energy resolution, thinner fluorescence films should be considered, with the drawback of losing quantum efficiency.

The photon energy-direction correlation is transformed into an energy-position correlation along the horizontal dimension of the CCD detector. The intensity along one horizontal line of pixels gives an XAS signal. With $20^{\circ}$ of incidence, the vertical beam ( $200 \mu \mathrm{m}$ FWHM) is spread over about 18 pixels. To produce an XAS frame, the XAS signal is integrated along the vertical dimension over 30 to 50 pixels. Each pixel in the CCD detector is an integrator. It accumulates charges that are transferred to a processing system. A pixel saturates with about $2.0 \times 10^{5}$ photons of $6 \mathrm{keV}$ (or, with about 275000 electrons). So, if $1.0 \times 10^{11}$ photons $\mathrm{s}^{-1}$, distributed within typically 1000 horizontal by 40 vertical pixels, reach the detector, each pixel receives on average $2.5 \times$ $10^{6}$ photons s ${ }^{-1}$. The pixels at the maximum of the Gaussian

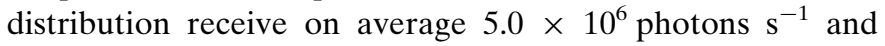
would saturate in about $40 \mathrm{~ms}$. Typical accumulation time for
XAS frames is 20 to $1000 \mathrm{~ms}$, depending on photon flux and sample attenuation. So, the intensity at each point in an XAS frame comes from $4.0 \times 10^{6}$ photons at most and the statistical noise would be $5.0 \times 10^{-4}$. Unfortunately, the noise level in the detector system is not coming from the counting statistics of photons; it is also dependent on charges from thermal activation, leaks and transfer processing. Beam fluctuations and instabilities along with sample inhomogeneities may also have an important impact on the noise level. The final XAS spectrum is the average of many frames, ensuring a good signal/noise ratio is achieved.

\subsection{The optical bench}

The $2 \theta$ optical bench (Fig. 7, see also Fig. 2) is made of a steel-framed $3 \mathrm{~m}$-long granite block, with two main steel rails screwed directly onto aluminium reference pads aligned on the granite block. The optical bench can bear heavy equipment and hold movable supports that are clamped to the steel rails through linear sliders. We should recall that the crystal monochromator and its internal mechanics are isolated from the vacuum chamber and supported directly in a concrete block on the floor. This makes access to the center of rotation of the monochromator difficult and imposes the design of a $2 \theta$ arm rotation based on two translation stages equipped with linear encoders, ball screws and linear bearings (Neuenschwander \& Tolentino, 2004). After a proper alignment procedure, a set of equations that control each translation stage displacement is found in order to give a rotation referenced to the center of the monochromator. The rotation covers the $2 \theta$ range from 16 to $56^{\circ}$. The translation stages are both perpendicular to the optical bench at an angle of $36^{\circ}$ (Fig. 2). The short stage (shown in Fig. 7) has the moving carriage equipped with a rotation platform that contains a pivoting base plate. The long stage (not seen in Fig. 7) has the

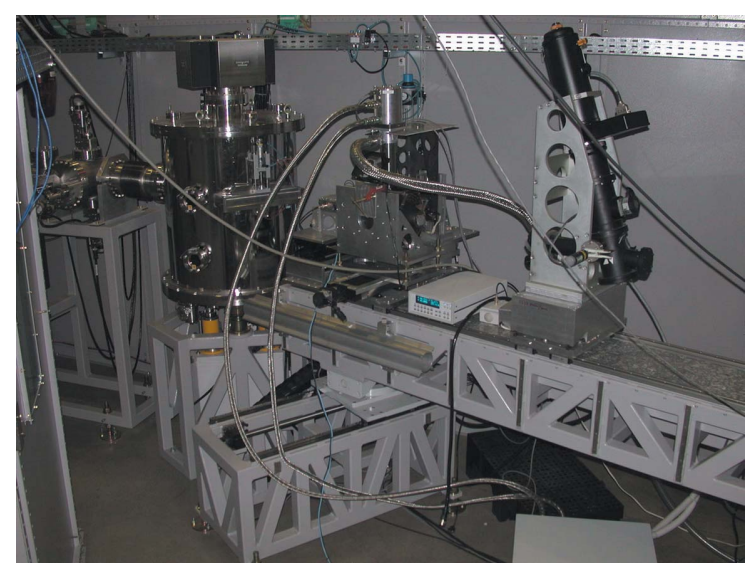

\section{Figure 7}

Photograph of the EDXAS beamline showing (from left to right) the entrance slits, the cylindrical monochromator vessel, with the large output Be window, the experimental set-up, with the cryostat and an electromagnet installed, and the modified CCD detector. The experimental setup and the detector are installed on the $2 \theta$ optical bench, which is supported on two linear stages (only one shortest linear stage appears in the photograph). 
moving carriage equipped with another linear stage able to move freely in the perpendicular direction. The $2 \theta$ arm is screwed onto the base plate of the short stage and placed by gravity in the center of an axial bearing at the top of the long stage. Recently, in order to receive the new $6.5 \mathrm{~T}$ superconducting magnet facility, the optical bench has been modified, keeping the same design but with the main rotatable arm much lower than the one shown here.

\section{Case study 1: XMCD in Cr-doped $\mathrm{LaMnO}_{3+\delta}$}

Doped $\mathrm{LaMnO}_{3}$ perovskites have been extensively studied owing to their colossal magneto-resistance (CMR) property and its potential applications to magnetic devices (Ramirez, 1997). In Cr-doped manganites the chromium takes the $\mathrm{Cr}^{3+}$ form. This ion involves three electrons in the one-spin state of the $t_{2 g}$ orbitals, analogous to $\mathrm{Mn}^{4+}$. For low doping concentrations, $\mathrm{Cr}^{3+}$ ions go into the $\mathrm{Mn}^{3+}$ sites, thereby changing the $\mathrm{Mn}^{3+}$ density without large distortion of the crystal cell (Morales et al., 2008). These features make the partial substitution of $\mathrm{Mn}$ by $\mathrm{Cr}$ in $\mathrm{LaMnO}_{3.00+\delta}$ an interesting system for studying the character of the $\mathrm{Mn}^{3+}-\mathrm{Cr}^{3+}$ interaction (Sun et al., 2001; Morales et al., 2005; Deisenhofer et al., 2002) and understanding the mechanism involved in CMR.

We present here a preliminary XMCD study in a series of $\mathrm{LaMn}_{1-x} \mathrm{Cr}_{x} \mathrm{O}_{3.00+\delta}$ compounds with rigorously controlled stoichiometry. Polycrystalline powdered samples of $\mathrm{LaMn}_{0.75} \mathrm{Cr}_{0.25} \mathrm{O}_{3.00}, \mathrm{LaMn}_{0.9} \mathrm{Cr}_{0.1} \mathrm{O}_{3.04}$ and $\mathrm{LaMnO}_{3.09}$ were prepared by the liquid-mix method (Morales et al., 2008). $\mathrm{X}$-ray powder diffraction reveals the existence of single-phase materials with orthorhombic symmetry (Pnma). With the increase of $\mathrm{Cr}$ content the magnetic phases change from Atype AFM order to a system with FM clusters. In sample A $\left(\mathrm{LaMn}_{0.75} \mathrm{Cr}_{0.25} \mathrm{O}_{3.00}\right)$ there are no $\mathrm{Mn}^{4+}$ ions, the $\mathrm{FM}$ ordering temperature is $90 \mathrm{~K}$ and the magnetic moment is $1.6 \mu_{\mathrm{B}}$. The holes $(25 \%)$ are all introduced by Cr-doping. The two other samples contain the same hole ratio (18\%). However, in sample $\mathrm{B}\left(\mathrm{LaMnO}_{3.09}\right)$ the holes are introduced only by the oxygen over-stoichiometry, then formally present as $\mathrm{Mn}^{4+}$ ions, while in sample $\mathrm{C}\left(\mathrm{LaMn}_{0.9} \mathrm{Cr}_{0.1} \mathrm{O}_{3.04}\right)$ half of them are provided by Cr-doping. The magnetic moments for samples $\mathrm{B}$ and $\mathrm{C}$ are $3.0 \mu_{\mathrm{B}}$ and $2.5 \mu_{\mathrm{B}}$ (Morales \& Caneiro, 2003), with ordering temperatures of $165 \mathrm{~K}$ and $135 \mathrm{~K}$ (Morales et al., $2004)$, respectively. At the Mn $K$ edge, the XMCD is due to the orbital magnetization of $4 p$-projected density of unoccupied states (Guo, 1998). These states are hybridized with the spinpolarized $3 d$ Mn band, which dominates the magnetic properties of the system. Thus the XMCD amplitude reflects the Mn magnetic moment strength.

XMCD spectra at the Mn and $\mathrm{Cr} K$ edges $(6539 \mathrm{eV}$ and $5989 \mathrm{eV}$, respectively, in the pure elements) were collected with an average storage ring current of $250 \mathrm{~mA}$. The circularly polarized light was selected $0.14 \mathrm{mrad}(0.8 \mathrm{~mm})$ above the orbital plane, with a slit aperture of $0.02 \mathrm{mrad}(100 \mu \mathrm{m})$. Under such conditions the photon flux reaching the sample was reduced to about $5.0 \times 10^{10}$ photons $\mathrm{s}^{-1}$ with an estimated polarization ratio of $70 \%$ (Tolentino et al., 2005a). A band- pass of $230 \mathrm{eV}$ with an energy resolution of $1.5 \mathrm{eV}$ was selected around the chosen edge by the $\mathrm{Si}(111)$ bent crystal. The beam was focused horizontally to a spot of $200 \mu \mathrm{m}$ and collimated vertically to about $100 \mu \mathrm{m}$. Data were collected using the CCD detector, with the sample temperature fixed at $20 \mathrm{~K}$ using a closed-cycle helium cryostat. The direction of the $0.9 \mathrm{~T}$ applied magnetic field was inverted by $180^{\circ}$ rotation of the permanent magnet. To avoid long-term shifts in energy scale, the sequence of collection followed the standard procedure with the magnetic field aligned parallel to the beam direction $(+)$, then antiparallel $(-)$, then again antiparallel $(-)$, then finally parallel $(+)$. The sequence is repeated starting with antiparallel, leading to the XMCD signal as the average of these eight (+--+-++-) acquisitions. The typical acquisition time for each XAS frame was $300 \mathrm{~ms}$. Each acquisition was the result of typically 50 accumulations, or about $15 \mathrm{~s}$. So, a total of $50 \times 10^{6}$ photons were accumulated at each acquisition, giving a statistical fluctuation of $1.4 \times 10^{-4}$. However, the noise in this experiment was not limited by the photon counting. Beam fluctuations and sample vibrations, induced by the rotation of the magnet, associated with sample inhomogeneities, were probably the main source of noise. Many successive acquisitions, typically 100 to 500 , were added up in order to obtain a noise-to-signal ratio of the order of $10^{-4}$.

Since the Cr $K$ and La $L_{2}$ edges are only $100 \mathrm{eV}$ apart (5989 eV and $5891 \mathrm{eV}$, respectively), the XANES features of the former are superimposed on the EXAFS (extended X-ray absorption fine structure) oscillations of the latter. Although $\mathrm{La}$ is a nominally diamagnetic ion, a strong XMCD signal at the La $L_{2}$ edge is clearly observed (Fig. 8). This signal is likely to arise from a ferromagnetic-induced moment by interactions with neighboring $\mathrm{Mn}$ ions. The $\mathrm{La} L_{2}$ background signal, extending over a large energy range far above the $\mathrm{Cr} K$ edge, is therefore not removed by performing differences among sequential spectra. The data presented in Fig. 8 correspond to the sample $\mathrm{A}$, with the higher $\mathrm{Cr}$ content, and an accumulated acquisition time of about $5 \mathrm{~h}$, giving a noise level of $1.5 \times 10^{-4}$, which represents the RMS statistics of the spectrum when it is

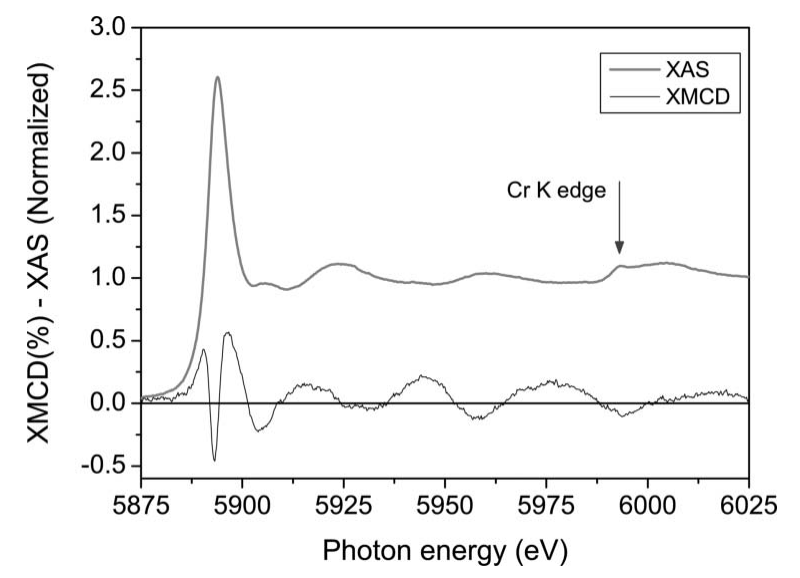

Figure 8

La $L_{2}$-edge and $\mathrm{Cr} K$-edge XANES (bold line) and XMCD (thin line) in sample $\mathrm{A}\left(\mathrm{LaMn}_{0.75} \mathrm{Cr}_{0.25} \mathrm{O}_{3.00}\right)$. The XANES features at the $\mathrm{Cr} K$ edge are superimposed on the $\mathrm{La} L_{2}$-edge EXAFS oscillations. Only the $\mathrm{La} L_{2}$ $\mathrm{XMCD}$ signal is detectable in these experiments. 
normalized to the $\mathrm{La} L_{2}$ edge. If the spectrum is normalized to the Cr $K$ edge, whose jump is about ten times smaller, the noise level is of the order of $10^{-3}$. Within such a noise level, no $\mathrm{Cr} K$-edge XMCD signal has been observed. As we will see below, the XMCD amplitude at the Mn $K$ edge, which refers to the amplitude of the first negative feature around $6553 \mathrm{eV}$, ranges from 1.4 to $2.5 \times 10^{-3}$. If $\mathrm{Cr}$ were coupled antiferromagnetically or ferromagnetically with $\mathrm{Mn}$, one would expect a value quite close to that of $\mathrm{Mn}$. We can conclude that either the $\mathrm{Cr}$ spins are randomly oriented and average out, or they give a contribution to the XMCD signal by less than $10^{-3}$. To be able to extract any signal smaller than that, at least one order of magnitude should be gained in the noise-to-signal ratio. In this experiment the noise was not limited by the counting statistics of photons but by a combination of source instabilities, inhomogeneities of the sample and sample holder vibrations. New dedicated measurements, with longer acquisition time, should significantly improve the noise-to-signal ratio.

The normalized XANES spectrum at the Mn $K$ edge, its first derivative and the $\mathrm{XMCD}$ signal in $\mathrm{LaMnO}_{3.09}$ (sample $\mathrm{B}$ ) are shown in Fig. 9 for a total acquisition time of $3 \mathrm{~h}$. The XMCD and the XANES derivative shapes are quite different. Some of their near-edge features are almost out of phase, testifying that the XMCD is not a derivative spurious signal. The XANES first derivative can be used to estimate the beamline stability required for detecting reliable XMCD signals. In the present case, the maximum derivative at the edge position is about $2.3 \times 10^{-4} \mathrm{meV}^{-1}$. This means that, in the procedure of difference among successive spectra, an instability in the energy scale of $1 \mathrm{meV}$ would give rise to a spurious contribution of $2.3 \times 10^{-4}$ and then hinder any expected XMCD contribution of same order of magnitude. The noise level of our XMCD data is $1.0 \times 10^{-4}$. Successive measurements of the same sample for several hours show a

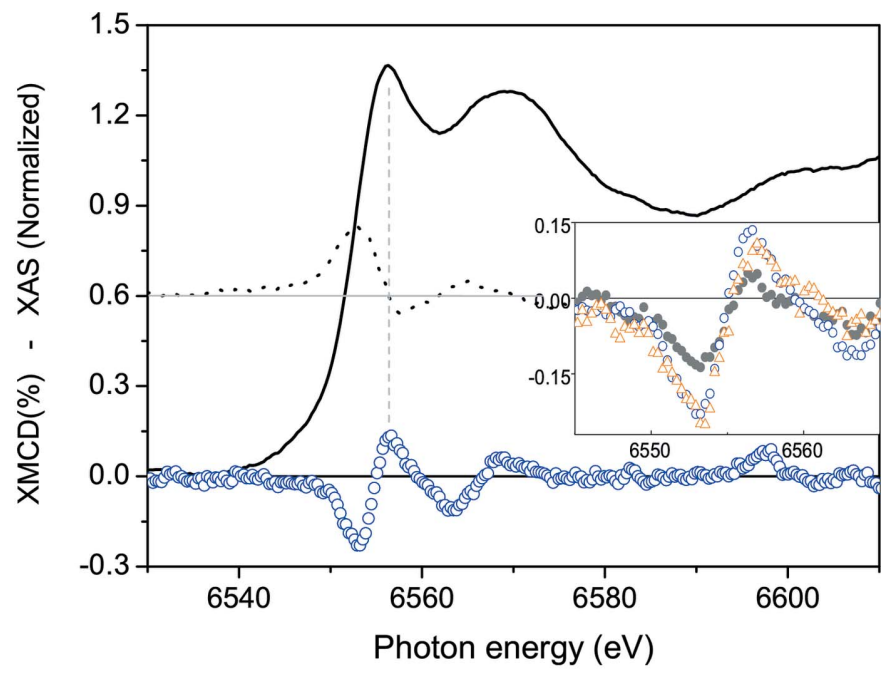

Figure 9

Mn $K$ edge in sample B $\left(\mathrm{LaMnO}_{3.09}\right)$ : XANES (plain line), derivative of the XANES (dotted line) and XMCD signal (open circles). Inset: XMCD signal for the three samples A (plain circles), B (open circles) and C (open triangles). variation smaller than $10 \%$ in signal amplitude, thus attesting an energy stability of the order of $1 \mathrm{meV}$. For the sample B, the Mn $K$-edge XMCD amplitude is $2.5 \times 10^{-3}$ (or $3.6 \times 10^{-3}$, if corrected by the polarization ratio of $70 \%$ ), in agreement with those found in doped manganites compounds with similar hole doping (Subias et al., 1997).

The XMCD signals of the different samples A, B and C (Fig. 9 and insert) have the same shape, already identified in doped manganites: a main signal changing from negative to positive at the edge, then a second resonance stemming from multiple scattering of magnetic nearest neighbors, and finally a further peak, observed at $43 \mathrm{eV}$ above the edge, assigned to a simultaneous excitation of $1 s$ and $3 p$ electrons (Subias et al., 1997). We note that the XMCD amplitudes are very similar for samples $B\left(2.5 \times 10^{-3}\right)$ and $C\left(2.3 \times 10^{-3}\right)$, and about half in amplitude for sample $\mathrm{A}\left(1.4 \times 10^{-3}\right)$. The lower amplitude in sample $\mathrm{A}$ is easily explained considering that the magnetic moment of this sample $\left(1.6 \mu_{\mathrm{B}}\right)$ is half that of sample $\mathrm{B}$ $\left(3.0 \mu_{\mathrm{B}}\right)$. This is consistent with the strong domination of the $3 d \mathrm{Mn}$ spins on the magnetic properties of manganites. More singular is the similarity of the XMCD signals for samples B and $\mathrm{C}$. While in the case of the mixed (hole plus Cr-)doped sample $\mathrm{C}$ the total magnetic moment is significantly $(20 \%)$ smaller $\left(2.5 \mu_{\mathrm{B}}\right)$ with respect to the strictly hole-doped sample B $\left(3.0 \mu_{\mathrm{B}}\right)$, the XMCD signals for both samples are the same within the accuracy of $10 \%$. The Mn atoms in both samples polarize the $4 p$ states in the same manner and then carry similar magnetic moments. A lower magnetic moment for sample $\mathrm{C}$ could be related to an AFM $\mathrm{Mn}^{3+}$ and $\mathrm{Cr}^{3+}$ spin alignment. Further measurements are needed to confirm and extend this assumption.

\section{Case study 2: pressure-induced metal-insulator transition in $\mathrm{YNiO}_{3}$}

Distorted nickel perovskites $R \mathrm{NiO}_{3}(R=\mathrm{Y}$ or rare-earth) exhibit a narrow thermally induced insulator-metal (IM) transition (except for La) (Lacorre et al., 1991). The transition temperature ( $\left.T_{\mathrm{IM}}\right)$ increases when the $R$ atomic size decreases. For the largest sizes $(R=\mathrm{Pr}, \mathrm{Nd}, \mathrm{Sm})$ diffraction measurements show an orthorhombic structure with a unique rather symmetric Ni site (García-Muñoz et al., 1992). For the smallest sizes $(R=\mathrm{Y}, \mathrm{Ho}-\mathrm{Lu})$ a monoclinic structure shows up below $T_{\mathrm{IM}}$. This monoclinic structure is characterized by the presence of two distinct $\mathrm{Ni}$ sites with different average $\mathrm{Ni}-\mathrm{O}$ distances, both exhibiting a limited distortion of the $\mathrm{NiO}_{6}$ octahedra (Alonso et al., 1999). EXAFS and XANES provided evidence for two Ni sites in the insulating phase even in the compound with orthorhombic structure (Piamonteze et al., 2005a; Acosta-Alejandro et al., 2008). The site with the longer $\mathrm{Ni}-\mathrm{O}$ distances is less hybridized and promotes electronic localization. Charge-transfer multiplet simulations of the modifications in the $\mathrm{Ni} L$-edge spectral shape for the insulating and metallic states undoubtedly confirmed this scenario (Piamonteze et al., 2005b).

The stability of the insulating charge-ordered state in $\mathrm{YNiO}_{3}$ under applied pressure was investigated by infrared 
spectroscopy and X-ray diffraction (García-Muñoz et al., 2004). The authors report a sudden structural modification close to $14 \mathrm{GPa}$, consistent with a monoclinic to orthorhombic transition. At this pressure an increase in electronic conductivity and a phonon screening were also observed. However, such structural modifications of long-range character could not be directly associated with the insulator-metal transition.

We report here on the evolution under pressure of the local scale environment of $\mathrm{Ni}^{3+}$ ions in $\mathrm{YNiO}_{3}$, directly studied by high-pressure XAS at the Ni $K$ edge $(8345 \mathrm{eV})$. A fine-grained high-quality $\mathrm{YNiO}_{3}$ polycrystalline powder sample (Alonso et al., 2000) was loaded into the $125 \mu \mathrm{m}$-diameter hole of an iconel gasket mounted on $2.4 \mathrm{~mm}$-thick diamond anvils. At $8 \mathrm{keV}$ such thickness results in an attenuation of $96 \%$ of the incident beam. In order to keep a similar dynamic range of the CCD detector, the normalization intensity without the sample was measured through an equivalent hole gasket and attenuated by thin glass plates. Each frame was collected in $100 \mathrm{~ms}$ and accumulated 250 times, giving a total acquisition time of $20 \mathrm{~s}$ per spectrum. Five spectra were collected at each pressure and then averaged to produce the final XAS data. XAS was measured with an energy resolution of about $1.5 \mathrm{eV}$, a bandpass of about $800 \mathrm{eV}$ being focused to a Gaussian spot of $130 \mu \mathrm{m}$ (FWHM). For each energy the center position was dispersed in less than $30 \mu \mathrm{m}$, in such a way that almost the whole energy band-pass could be collected through the gasket. Quasi-hydrostatic pressures up to $20 \mathrm{GPa}$ were applied using silicone oil as pressure medium. The actual pressure was increased by steps of about $2 \mathrm{GPa}$, monitored and controlled with a precision of about $0.5 \mathrm{GPa}$, using the luminescence from ruby spheres. After each pressure measurement, the cell was carefully realigned at the optical focus. The energy calibration stability was better than $0.03 \mathrm{eV}$ over the whole set of measurements.

Fig. 10 shows the Ni $K$-edge XANES spectra of $\mathrm{YNiO}_{3}$ at selected pressures. Two main changes are observed when increasing applied pressure: a shift of the absorption threshold

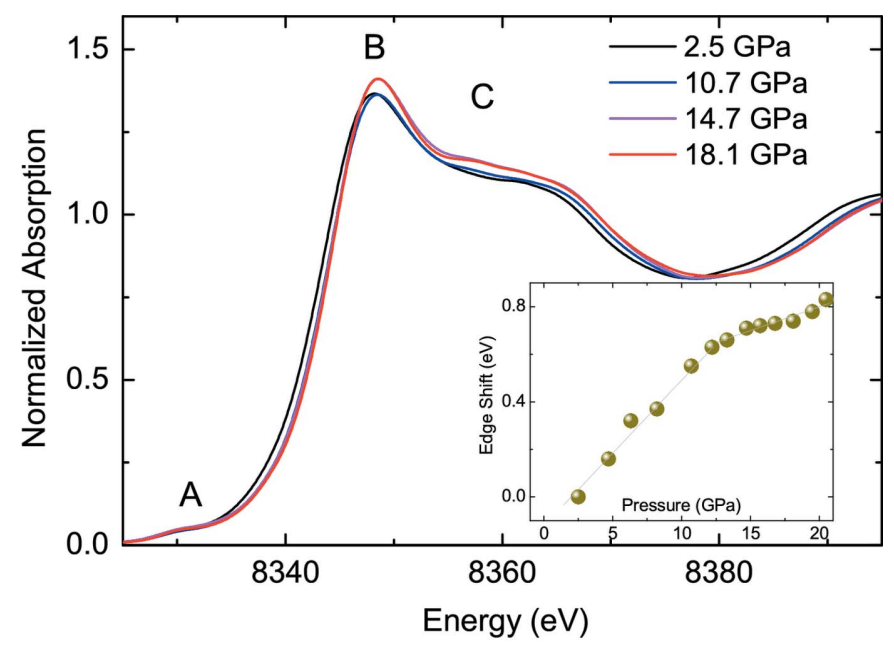

Figure 10

Comparison of the XANES spectra in $\mathrm{YNiO}_{3}$ at selected applied pressures. Inset: the edge energy position shift for applied pressures. and a subtle raise of the structures $\mathrm{B}$ and $\mathrm{C}$ above the edge; the feature A does not change. The absorption threshold shifts almost continuously towards higher energies. In order to properly interpret this shift, the reproducibility of the edge position was checked to be better than $30 \mathrm{meV}$. The accuracy can also be certified by the fact that feature A does not change and that after pressure release the features are rather recovered. The amplitude of the edge shift as a function of the increment of applied pressure, $\delta E / \delta P$, is not constant over the whole pressure range (insert Fig. 10). Up to about $13 \mathrm{GPa}, \delta E$ varies almost linearly with the applied pressure and quotes about $60 \mathrm{meV} \mathrm{GPa}^{-1}$. Above that, $\delta E / \delta P$ drops to $20 \mathrm{meV} \mathrm{GPa}^{-1}$ and another almost linear regime takes place. As the metal formal valence $\left(\mathrm{Ni}^{3+}\right)$ does not change with the applied pressure, the edge shift $(\delta E)$ results essentially from the modifications in the repulsive nearest-neighbor potential arising from the bond reduction $(\delta R)$ in the coordination shell. As for other perovskite oxides, where the ratio $\delta E / \delta R$ is roughly $10 \mathrm{eV}^{-1}$ (Ramos et al., 2007), the shift may be converted directly into a compression. The compression separated in the two different linear regimes turns out to be $\sim 0.06 \AA$ and $0.02 \AA$, respectively. It should be noted that the difference in the average distance of the two $\mathrm{Ni}$ sites in the monoclinic structure is $\sim 0.08 \AA$.

A subtle rise in the intensity of the structures $\mathrm{B}$ and $\mathrm{C}$ above the edge (from 8350 to $8370 \mathrm{eV}$ ) occurs in a narrow pressure range from 12 to $14 \mathrm{GPa}$. The sudden increase of the spectral weight of the B structure is associated with some degree of symmetrization in the average Ni site (Ramos et al., 2007). The rise of the $\mathrm{C}$ structure has been observed in the thermal evolution of the $\mathrm{PrNiO}_{3}$ compound across the insulator-metal transition (Acosta-Alejandro et al., 2008). It has unambiguously been associated with a local symmetry change from two non-equivalent $\mathrm{Ni}$ sites, consistent with a local monoclinic distorted structure, to a single rather symmetric Ni site.

The EXAFS oscillations, given in Fig. 11 for selected applied pressures, clearly show the good quality of the signal, free of spurious diffraction peaks from the diamond anvil cell,

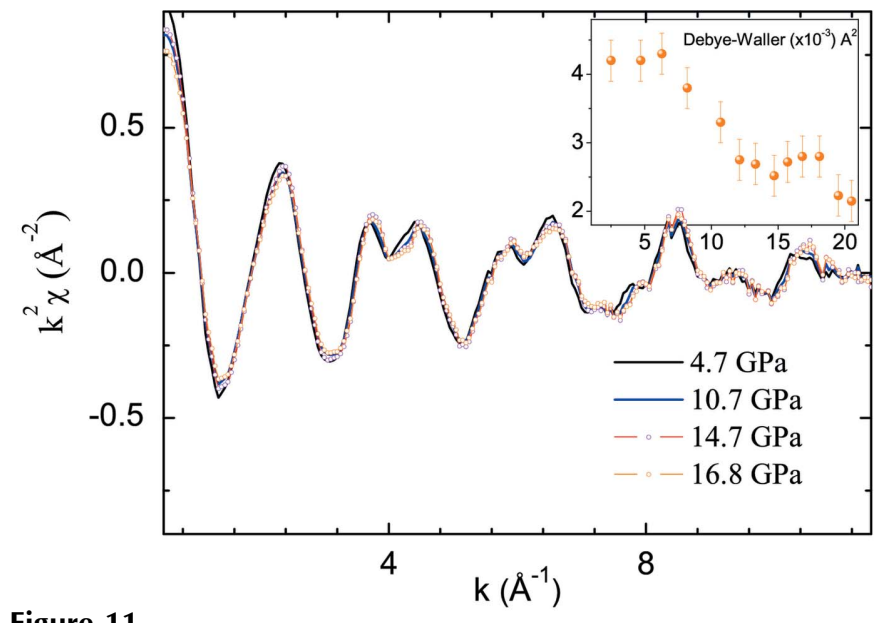

Figure 11

Comparison of $k^{2}$-weighted EXAFS oscillations of $\mathrm{YNiO}_{3}$ at selected pressures around $13 \mathrm{GPa}$. Insert: Debye-Waller term variation as a function of applied pressure. 
over a large extension in reciprocal space $\left(k_{\max } \simeq 12 \AA^{-1}\right)$. When the applied pressure increases, the overall structure of the oscillations remains almost unchanged but their amplitude slightly increases. Such a feature is usually associated with a decrease in the damping Debye-Waller (DW) term $\exp \left(-2 k^{2} \sigma^{2}\right)$ accounting for bond length dispersion. The DW variation related to the coordination shell (inset Fig. 11) decreases almost linearly from 6 to $14 \mathrm{GPa}$. This decrease is in agreement with the reduction in the splitting of $\mathrm{NiO}$ distances. A complete EXAFS analysis must be carried out to quantify this decrease. A similar drop was observed at the crossover of the thermally induced insulator-metal transition in $\mathrm{NdNiO}_{3}$ (Piamonteze et al., 2005a).

All local experimental evidence coming from XAS suggests a coherent description of the pressure-induced process leading to the insulator-metal transition in $\mathrm{YNiO}_{3}$ at room temperature. In a first regime of compression, the largest distorted $\mathrm{Ni}$ site shrinks, while the smallest one remains almost unchanged, until symmetrization at the critical pressure of $\sim 13 \mathrm{GPa}$. At this pressure all $\mathrm{NiO}_{6}$ octahedra then become roughly equivalent, charge delocalization takes place and the system becomes metallic at room temperature. The additional pressure effect is limited to an overall bond length reduction, with a decreased compressibility.

\section{Summary}

An overview of the EDXAS beamline implemented at the Brazilian Synchrotron Light Laboratory has been given. The choice of the optics and detection scheme has been determined by the bending-magnet source characteristics. The optics, mirror and monochromator, very close to the source, have the advantage that a large angular beam acceptance can be collected by the monochromator. Moreover, a few number of optical elements and short distances are favorable to produce a very stable beam. The large source size and a maximum demagnification of the order of 10 impose the size of the focused beam, which is typically $250 \mu \mathrm{m} \times 200 \mu \mathrm{m}$. The focus dimension is not limited by aberrations from the bent crystal but by the source size. This focus is quite large compared with facilities installed at higher-energies/loweremittances synchrotron sources. The ODE beamline at Soleil utilizes a similar optics to produce a focus of $40 \mu \mathrm{m} \times 40 \mu \mathrm{m}$. At ID24 at ESRF, the beam size can be as small as $5 \mu \mathrm{m} \times$ $5 \mu \mathrm{m}$, which opens the possibility of micrometer mapping of inhomogeneous samples. A modified CCD detector that uses a fluorescence screen to convert X-rays into visible photons has been optimized to match the photon flux conditions. Typical acquisition times from $10 \mathrm{~ms}$ to $500 \mathrm{~ms}$ are achieved in most experiments.

In this paper two scientific cases related to strongly correlated metal oxides have been shown. In the first case, the magnetic properties of Cr-doped manganites were investigated by XMCD experiments. The preliminary results suggest that at least part of $\mathrm{Cr}$ spins are randomly distributed within the system or antiferromagnetically coupled to $\mathrm{Mn}^{3+}$ spins. These results demonstrate the feasibility of experiments on diluted light metal elements in strongly absorbing compounds. In a second case study, a high-pressure diamond anvil cell was used to induce the metallic phase in $\mathrm{YNiO}_{3}$ at room temperature. The insulator-metal transition of $\mathrm{YNiO}_{3}$ under normal atmospheric conditions is $580 \mathrm{~K}$ and the effect of pressure is to reduce the structural distortion, decreasing the transition temperature. Much experimental evidence has been gathered providing a coherent local structural description across the IM transition. The stable $250 \mu \mathrm{m} \times 200 \mu \mathrm{m}$ beam matches very well the diamond anvil cell for experiments in the pressure range up to $20 \mathrm{GPa}$.

The EDXAS beamline has proved to be a valuable tool for a variety of material science studies. The instrumentation has been used to investigate magnetic materials, via XMCD experiments, electrochemical and catalytic reactions and phase transitions under pressures up to $20 \mathrm{GPa}$, covering the fields of similar existing EDXAS facilities around the world. Owing to the LNLS storage ring characteristics, which are mostly a soft X-ray machine with a critical energy of $2.08 \mathrm{keV}$, the energy range covered by the beamline, from 4 to $14 \mathrm{keV}$, suffers from a limited photon flux. Nevertheless, the fluorescence screen was optimized to gain in quantum efficiency and this is not the most serious limitation. The quite large emittance, however, imposes a focused beam of $150 \mu \mathrm{m}$ (FWHM), or $250 \mu \mathrm{m}$ (full beam), and limits the highest attainable pressure using diamond anvil cells. It can also be a dramatic limitation when samples are inhomogeneous. On the other hand, the possibility of being very close to the source and the simplicity of the optics, with few optical elements, leads to a good stability over long periods.

We acknowledge the LNLS technical staff. Special thanks to Milton Rocha for providing technical drawings. This work is partially supported by LNLS/ABTLuS/MCT and FAPESP (1999/12330-6). JCC, NMSN and AYR acknowledge the grants from FAPESP, CAPES and CNPq, respectively.

\section{References}

Acosta-Alejandro, M., Mustre de León, J., Medarde, M., Lacorre, P., Konder, K. \& Montano, P. (2008). Phys. Rev. B, 77, 085107.

Alonso, J. A., García-Muñoz, J. L., Fernández-Díaz, M. T., Aranda, M. A. G., Martínez-Lope, M. J. \& Casais, M. T. (1999). Phys. Rev. Lett. 82, 3871-3874.

Alonso, J. A., Martínez-Lope, M. J., Casais, M. T., García-Muñoz, J. L. \& Fernández-Díaz, M. T. (2000). Phys. Rev. B, 61, 1756-1763.

Azimonte, C., Cezar, J., Granado, E., Huang, Q., Lynn, J., Campoy, J., Gopalakrishnan, J. \& Ramesha, K. (2007). Phys. Rev. Lett. 98, 017204.

Baudelet, F., Dartyge, E., Fontaine, A., Brouder, C., Krill, G., Kapler, J. P. \& Piecuch, M. (1991). Phys. Rev. B, 43, 5857-5866.

Bernardi, F., Alves, M., Scheeren, C., Dupont, J. \& Morais, J. (2007). J. Electron Spectrosc. Relat. Phenom. 156-158, 186-190.

Bernardi, F., Alves, M. C. M., Traverse, A., Silva, D. O., Scheeren, C. W., Dupont, J. \& Morais, J. (2009). J. Phys. Chem. C, 113, 39093916.

Bonfim, M., Mackay, K., Pizzini, S., San Miguel, A., Tolentino, H., Giles, C., Neisius, T., Hagelstein, M., Baudelet, F., Malgrange, C. \& Fontaine, A. (1998). J. Synchrotron Rad. 5, 750-752. 
Dartyge, E., Depautex, C., Dubuisson, J. M., Fontaine, A., Jucha, A., Leboucher, P. \& Tourillon, G. (1986). Nucl. Instrum. Methods Phys. Res. A, 246, 452-460.

Deisenhofer, J., Paraskevopoulos, M., Krug von Nidda, H.-A. \& Loidl, A. (2002). Phys. Rev. B, 66, 054414.

Ferreira, F. F., Correa, H. P. S., Orlando, M. T. D., Passamai, J. L., Orlando, C. G. P., Cavalcante, I. P., Garcia, F., Tamura, E., Martinez, L. G., Rossi, J. L. \& de Melo, F. C. L. (2009). J. Synchrotron Rad. 16, $48-56$.

García-Muñoz, J., Amboage, M., Hanfland, M., Alonso, J. A., Martinez-Lope, M. J. \& Mortimer, R. (2004). Phys. Rev. B, 69, 094106.

García-Muñoz, J. L., Rodríguez-Carvajal, J., Lacorre, P. \& Torrance, J. B. (1992). Phys. Rev. B, 46, 4414-4425.

Godoi, D. R. M., Perez, J. \& Villullas, H. M. (2009). J. Phys. Chem. C, 113, 8518-8525.

Guo, G. Y. (1998). Phys. Rev. B, 57, 10295-10298.

Itie, J. P., Polian, A., Calas, G., Petiau, J., Fontaine, A. \& Tolentino, H. (1989). Phys. Rev. Lett. 63, 4-8.

Koch, A. (1994). Nucl. Instrum. Methods Phys. Res. A, 348, 654-658.

Lacorre, P., Torrance, J. B., Pannetier, J., Nazzal, A. I., Wang, P., Huang, T. \& Siemens, R. (1991). J. Solid State Chem. 91, 225-237.

Lin, L., Tavares, P. F. \& Tosin, G. (2001). IEEE Proc. 5, 2701.

Mathon, O., Baudelet, F., Itié, J.-P., Pasternak, S., Polian, A. \& Pascarelli, S. (2004). J. Synchrotron Rad. 11, 423-427.

Meneses, C., Flores, W. \& Sasaki, J. (2007). Chem. Mater. 19, 10241027.

Meneses, C. T., Flores, W. H., Sotero, A. P., Tamura, E., Garcia, F. \& Sasaki, J. M. (2006). J. Synchrotron Rad. 13, 468-470.

Morales, L., Allub, R., Alascio, B., Butera, A. \& Caneiro, A. (2005). Phys. Rev. B, 72, 132413.

Morales, L. \& Caneiro, A. (2003). J. Solid State Chem. 170, 404-410.

Morales, L., Rodriguez-Carvajal, J. \& Caneiro, A. (2004). J. Alloys Compd. 369, 97-100.

Morales, L., Zysler, R. \& Caneiro, A. (2008). J. Solid State Chem. 181, 1824-1832.

Neuenschwander, R. T. \& Tavares, W. S. (2001). Internal Technical Communication. Internal Technical Note, LNLS, Campinas, SP, Brazil.
Neuenschwander, R. T. \& Tolentino, H. C. N. (2004). AIP Conf. Proc. 705, 647-650.

Pascarelli, S., Mathon, O. \& Aquilanti, G. (2004). J. Alloys Compd. 362, 33-40.

Pettifer, R. F., Mathon, O., Pascarelli, S., Cooke, M. D. \& Gibbs, M. R. J. (2005). Nature (London), 435, 78-81.

Phizackerley, R. P., Rek, Z. U., Stephenson, G. B., Conradson, S. D., Hodgson, K. O., Matsushita, T. \& Oyanagi, H. (1983). J. Appl. Cryst. 16, 220-232.

Piamonteze, C., de Groot, F. M. F., Tolentino, H. C. N., Ramos, A. Y., Massa, N. E., Alonso, J. A. \& Martínez-Lope, M. J. (2005a). Phys. Rev. B, 71, 020406.

Piamonteze, C., Tolentino, H. C. N., Ramos, A. Y., Massa, N. E., Alonso, J. A., Martínez-Lope, M. J. \& Casais, M. T. (2005b). Phys. Rev. B, 71, 012104.

Pizzini, S., Fontaine, A., Giogetti, C., Dartyge, E., Bobo, J.-F., Piecuch, M. \& Baudelet, F. (1995). Phys. Rev. Lett. 74, 1470-1473.

Ramirez, A. (1997). J. Phys. Condens. Matter, 9, 8171-8199.

Ramos, A. Y., Tolentino, H. C. N., Souza-Neto, N. M., Itié, J. P., Caneiro, A. \& Morales, L. (2007). Phys. Rev. B, 75, 052103.

Rodrigues, A. R. D., Craievich, A. F. \& Gonçalves da Silva, C. E. T. (1998). J. Synchrotron Rad. 5, 1157-1161.

Souza-Neto, N. M., Ramos, A. Y., Tolentino, H. C. N., Martins, A. \& Santos, A. D. (2006). Appl. Phys. Lett. 89, 111910.

Stewart, S., Figueroa, S., Sturla, M., Scorzelli, R., Garcia, F. \& Requejo, F. (2007). Physica B, 389, 155-158.

Subias, G., Garcia, J., Proietti, M. \& Blasco, J. (1997). Phys. Rev. B, 56, 8183-8191.

Sun, Y., Tong, W., Xu, X. \& Zhang, Y. (2001). Phys. Rev. B, 63, 174438.

Tolentino, H. C. N., Cezar, J. C., Souza-Neto, N. M. \& Ramos, A. Y. (2005a). J. Synchrotron Rad. 12, 168-176.

Tolentino, H. C. N., Cezar, J. C., Watanabe, N., Piamonteze, C., SouzaNeto, N. M., Tamura, E., Ramos, A. \& Neueschwander, R. (2005). Phys. Scr. T115, 977-979.

Tolentino, H., Dartyge, E., Fontaine, A. \& Tourillon, G. (1988). J. Appl. Cryst. 21, 15-22.

Tourillon, G., Dartyge, E., Fontaine, A. \& Jucha, A. (1986). Phys. Rev. Lett. 57, 603-606. 